\title{
L'histoire au tribunal. Le cas des procès Touvier et Papon en France
}

En France, les procès PAPON en 19971998 et TOUVIER en 1994 ont reposé la question du bienfondé de l'intervention historienne dans le prétoire. Certains, comme Henry Rousso, ont souligné le
L'auteur : Thomas RIBÉMONT est maître de conférences en science politique à l'Université Paris 13-Sorbonne. risque qu'il y avait de voir le discours des historiens instrumentalisé au gré de la "rhétorique judiciaire " et/ou de voir se substituer la logique de justice à la démarche historienne. Comme nous le rappelions dans un précédent article, aux yeux de l'actuel directeur de l'Institut d'Histoire du Temps Présent (IHTP), le processus judiciaire "impose trop souvent aux historiens de répondre sur le mode de l'imputation (réponses binaires de type oui/non) à des questions dont ils ne sont pas maîtres. Or, selon lui, les règles de la méthode historique supposent l'autonomie du questionnement scientifique et prohibent le recours aux jugements d'imputation au profit de la recherche de causes multiples (...) $»^{2}$. D'autres historiens, tels Robert O. PAXTON, ou encore René RÉMOND, ont en revanche considéré que leur connaissance de la période couvrant les faits incriminés pouvait, malgré la dimension hétéronome ${ }^{3}$ de leur participation, servir l'œuvre de justice. Pour ces derniers, la demande d'histoire, qu'elle émane du judiciaire, des Etats, ou d'autres acteurs (minorités, associations...) ne doit pas être écartée par les historiens. "II n'est ni possible, ni souhaitable, écrivait René RÉMOND, que l'historien récuse totalement cette sorte de demande, parce qu'il ne peut tout à fait s'abstraire de son temps ni se soustraire complètement à la société dont il fait partie $»^{4}$. Pour les uns, la compétence spécifique de l'historien, fondée sur une méthodologie et des modalités d'explication visant à saisir des causalités

\footnotetext{
' Rousso Henry, La hantise du passé, Paris, Ed. Textuel, 1998, p. 103.

${ }^{2}$ RIBÉMONT Thomas, "Le procès PAPON : un cas d'expertise historienne ? ", in DAMAMME Dominique et RIBÉMONT Thomas, Expertise et engagement politique, Paris, L'Harmattan, collection " Les cahiers politiques », 2001, p. 73. Voy. aussi, sur cette séquence, FLEURY Béatrice et WALTER Jacques, "Le procès PAPON : médias, témoin-expert et contre-expertise historiographique ", Vingtième Siècle. Revue d'histoire, n 88 , 2005, pp. 63-76.

${ }^{3}$ Cette notion définit ici l'état d'un agent qui se soumet aux questions d'un tiers.

${ }^{4}$ RÉMOND René (dir.), Être historien aujourd'hui, UNESCO, Paris / Toulouse, Ed. Erès, 1988, p. 10.
} 
complexes, se révèle donc difficilement compatible avec les stratégies développées par les acteurs de l'enceinte judiciaire. Pour les autres, a contrario, la compétence professionnelle doit permettre, lorsque l'occasion se présente, de "faire la vérité " et "d'éclairer l'opinion lorsque celle-ci est troublée $n^{5}$.

Entre les tenants de ces diverses positions, les échanges ont été parfois d'autant plus vifs que, sur le fond, la discussion ne se limite pas au seul cadre judiciaire, mais achoppe plus largement sur la question des rapports que les historiens entretiennent avec la demande sociale et avec la pratique de l'expertise. D'aucuns, à l'instar de Gérard NOIRIEL, ont ainsi vu dans la montée en puissance des pratiques d'expertise la marque d'une transformation du rôle social de la discipline $^{6}$. Avec la reconnaissance du «devoir de mémoire », les historiens ${ }^{7}$ auraient été conduits à investir des problèmes intéressant directement la mémoire collective, ce qui reviendrait à accepter d'entrer dans des luttes politiques divisant des producteurs concurrents de discours sur le passé.

Suivant ce mouvement, les historiens ont notamment été amenés à déplacer le centre de gravité de leurs pratiques vers la scène judiciaire ${ }^{8}$, contribuant ainsi à une hybridation croissante entre l'espace du droit et de la recherche en histoire et à une politisation du savoir historien.

Ce processus d'hybridation renvoie en premier lieu à la juridicisation et à la judiciarisation des enjeux sociaux et politiques, évolution à laquelle n'échappe pas la discipline historique. Il est lié, par ailleurs, à la scénographie judiciaire elle-même, le statut des historiens dans une cour d'assises rendant difficile le cours de la réflexion historique. C'est pourquoi nombre de représentants de la discipline se sont demandés comment, dans un tel cadre, la connaissance historique pouvait concourir à la manifestation de la vérité et ne pas être instrumentalisée à des fins politiques.

\footnotetext{
${ }^{5}$ RÉmond René, AzÉma Jean-Pierre, BÉdarida François, Cholvy Gérard, Comte Bernard, Dujardin Jean, DURAND Jean-Dominique et HILAIRE Yves-Marie, Paul Touvier et l'Eglise. Rapport de la commission historique instituée par le cardinal Decourtray, Paris, Fayard, 1992, p. 13.

${ }^{6}$ NOIRIEL Gérard, Les origines républicaines de Vichy, Paris, Fayard, 1999.

${ }^{7}$ En particulier, selon Gérard NOIRIEL, les historiens du temps présent.

${ }^{8}$ Voy. par exemple sur ce point, Dumoulın Olivier, Le rôle social de l'historien. De la chaire au prétoire, 


\section{L'histoire, entre juridicisation et judiciarisation}

Dans une économie des rapports sociaux qui, depuis les années 1970, tend à épouser la logique judiciaire, les cadres d'exercice du métier d'historien, et spécifiquement de sa pratique experte, se déplacent. Cette évolution suscite de nouvelles contraintes, relatives, notamment, à l'instrumentalisation du discours historien, à son encadrement par le droit et au développement d'une «pensée de la contrebande $"^{9}$ que traduisent l'importation de catégories juridiques dans le champ de la recherche historique et la reproduction des normes symboliques de l'espace judiciaire dans les débats historiens.

Certes, la question des liens qui s'établissent entre la sphère judiciaire et l'histoire n'est, en tant que telle, pas nouvelle. Carlo GINZBURG ${ }^{10}$ en a largement fait état, rappelant notamment qu'au XVII siècle, déjà, la tradition classique rapprochait l'historien de l'avocat dont le but était d'emporter l'intime conviction des juges", non par l'administration de la preuve, mais par une argumentation qui puisse donner l'illusion de la réalité'2. De même, en 1769, dans son Traité des différentes sortes de preuves qui servent à établir la vérité historique ${ }^{13}$, Henri GRIFFET comparait l'historien à un juge passant au crible preuves et témoignages. En France, la synthèse entre ces deux approches (historien/avocat; historien/juge) s'opère d'ailleurs à la fin du XVII siècle, dans un contexte marqué par la domination des paradigmes positivistes, et par l'attention que les historiens portent à l'événement et aux individus.

De la fin du XIXe siècle aux premières décennies du XX $x^{e}$ siècle, écrit Carlo GINZBURG, I'historiographie, surtout l'historiographie politique - et, de façon toute particulière, I'historiographie de la Révolution française - prit un aspect judiciaire marqué. [...] Alphonse AULARD [...], tout comme Albert Mathiez, son adversaire à l'Université [revêtirent] tour à tour les habits de procureur de la République ou d'avocat de la défense pour prouver, en se fondant sur des "dossiers " circonstanciés, les responsabilités de Robespierre ou la corruption de DANTON. Cette tradition de réquisitoires à la fois politiques et moraux, suivis

\footnotetext{
- Voy. SIMÉANT Johanna, «Friches, Hybrides et contrebandes : sur la circulation et la puissance militantes des discours savants », in Hamman Philippe, MÉON Jean-Matthieu et VerRIER, Benoît (dir.), Discours savants, discours militants : mélange des genres, Paris, L'Harmattan, 2002, pp. 17-53.

10 Ginzburg Carlo, Le juge et l'historien. Considérations en marge du procès Sofri, Paris, Verdier, 1997.

"Voy. aussi sur ce point, CARbonnel Charles-Olivier, L'historiographie, Paris, Presses Universitaires de France, 1995.

${ }_{12}$ Procédé rhétorique classique mis en évidence par Roland Barthes à propos du roman et de la nouvelle, voy. sur ce point GENETTE Gérard et TODOROv Tzvetan (dir.), Littérature et réalité, Paris, Le Seuil, 1982.

${ }^{13}$ GRIFFET Henri, Traité des différentes sortes de preuves qui servent à établir la vérité historique, Liège, 
de condamnations ou d'acquittements, s'est poursuivie longtemps: Un jury pour la Révolution, écrit par un des célèbres historiens actuels de l'époque révolutionnaire, Jacques GODECHOT, fut publié en $1974^{14}$.

Cette tradition d' " historiographie judiciaire " ${ }^{15}$, attachée notamment à la Révolution française, et dont les résonances, comme le montre Reinhart KOSELLECK ${ }^{16}$, se font encore sentir aujourd'hui, n'a cependant pas manqué de susciter des interrogations et des remises en cause au sein de la profession historienne. Les premiers promoteurs des Annales, "en privilégiant l'histoire d'en bas sur l'histoire d'en haut, les groupes et les mentalités sur les individus, la longue durée sur l'événement $\aleph^{17}$, ont en particulier reproché au modèle judiciaire de conduire les historiens à attacher une trop grande attention aux événements tout en négligeant les phénomènes sociaux inscrits dans le temps long qui ne se prêtaient pas à un système d'explication rapportant les faits étudiés aux seules actions d'un ou de plusieurs individus ${ }^{18}$. En dépit des similitudes entre le travail du juge et celui de l'historien, Marc BLOCH réaffirmait ainsi la divergence des finalités recherchées:

\begin{abstract}
Un moment vient [...] où les chemins se séparent. Quand le savant a observé et expliqué, sa tâche est finie. Au juge, il reste encore à rendre sa sentence. Imposant silence à tout penchant personnel, la prononce-t-il selon la loi, il s'estimera impartial. Il le sera, en effet, au sens des juges. Non au sens des savants. Car on ne saurait condamner ou absoudre sans prendre parti pour une table de valeurs, qui ne relève plus d'aucune science positive. Qu'un homme ait tué un autre est un fait, éminemment susceptible de preuve. Mais châtier le meurtrier suppose qu'on tient le meurtrier pour coupable : ce qui n'est, à tout prendre, qu'une opinion sur laquelle toutes les civilisations ne sont pas tombées d'accord"19.
\end{abstract}

\footnotetext{
${ }^{14}$ Ginzburg Carlo, Le juge et l'historien, op. cit.., p. 18-19.

${ }^{15}$ La formule est empruntée à Luigi Ferrajoli. Voy. sur ce point, FERRAJOLI Luigi, Dritto et rapgione : theoria del garantisme penale, Roma, Editions Laterza, 1989.

${ }^{16}$ Réfléchissant sur les formes de justice intrinsèques à l'histoire des historiens, Reinhart Koselleck distingue cinq formes types : la réponse hérodotéenne, le modèle thycididéen, le modèle augustinien, l'histoire absurde et, reprenant la formule de SCHILLER, «l'histoire mondiale [comme] jugement du monde », KOSELLECK Reinhart L'expérience de l'histoire, Paris, Gallimard, 1997, pp. 161-181.

${ }^{17}$ LE CROM Jean-Pierre et MARTIN Jean-Clément, "Présentation », Droit et société, n³8, 1998, n "Vérité historique, vérité judiciaire ", p. 9 (9-11)

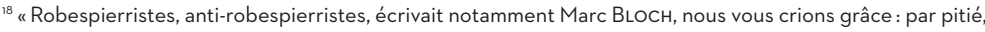
dites-nous seulement quel fut Robespierre ", BLOCH Marc, Apologie pour l'histoire ou Métier d'historien, Paris, Armand Colin, 1993, p. 157

19 Ibid., p. 156.
} 
Pour l'auteur des Rois Thaumaturges ${ }^{20}$, l'opération historique ne visait donc pas tant à juger qu'à comprendre ${ }^{21}$.

En outre, dans le dernier tiers du $x x^{e}$ siècle, de nombreux historiens ont été amenés à critiquer la notion de preuve ainsi que celle de vérité qui lui est intimement corrélée. L'après 1968 est, en effet, marqué par l'émergence de questionnements relatifs au problème du récit en histoire. Le linguistic turn américain, dont Hayden WHITE constitue la figure emblématique, en appliquant aux écrits historiques les apports de la psychanalyse, de la linguistique et de la sémiotique, a notamment cherché à inscrire l'histoire dans le répertoire de la fiction ${ }^{22}$. Pour les tenants de ce courant théorique, l'historien qui prétend avoir vu des archives ne fait, en définitive, que mettre en œuvre un procédé rhétorique. Dans cette perspective, les historiens ne construiraient pas un savoir que d'autres pourraient utiliser mais généreraient uniquement un discours sur le passé, le fait n'ayant dans cette perspective - déjà formalisée par Roland BARTHES - qu'une existence linguistique ${ }^{23}$.

En France, cette approche, qui tend à induire l'impossibilité de connaître la réalité, a trouvé un écho relativement important. Paul VEYNE, par exemple - voire, dans une moindre mesure, Michel DE CERTEAU ${ }^{24}$-, soutient que toute histoire est un récit événementiel, un « roman vrai » dans lequel l'explication «n'est guère que la manière qu'a le récit de s'organiser en une intrigue compréhensible $»^{25}$ :

L'histoire est un récit d'événements : tout le reste en découle. Puisqu'elle est d'emblée un récit, elle ne fait pas revivre, non plus que le roman; le vécu tel qu'il ressort des mains de l'historien n'est pas celui des acteurs; c'est une narration, ce qui permet d'éliminer certains

\footnotetext{
${ }^{20} \mathrm{BLOCH}$ Marc, Les rois thaumaturges. Etude sur le caractère surnaturel attribué à la puissance royale particulièrement en France et en Angleterre, Paris, Gallimard, 1993.

${ }^{21}$ "Le problème de l'utilité de l'histoire, au sens étroit, au sens " pragmatique » du mot utile, écrit-il, ne se confond pas avec celui de sa légitimité intellectuelle. II ne peut, d'ailleurs, venir qu'en second : pour agir raisonnablement, ne faut-il pas d'abord comprendre? ", BLOCH Marc, Les rois thaumaturges. Etude sur le caractère surnaturel attribué à la puissance royale particulièrement en France et en Angleterre, op.cit., p. 73. Au demeurant, à l'égard de circonstances exceptionnelles, le travail de compréhension, au sens de l'historien, ne va pas de soi. Primo Lévi le rappelait avec force : "Comprendre la décision ou la conduite de quelqu'un, cela veut dire (et c'est aussi le sens étymologique du mot) les mettre en soi, mettre en soi celui qui en est responsable. Eh bien, aucun être normal ne pourra s'identifier à HITLER, à HIMMLER, à GOEBBELS, à EICHMANN, à tant d'autres encore ", LeVı Primo, Si c'est un homme, Paris, Julliard, 1987, p. 211.

${ }_{22}$ Voy. notamment sur ce point, WHITE Hayden, Metahistory. The historical Imagination in Nine-teenthCentury Europe, Baltimore-London, The John Hopkins Press, 1973.

${ }^{23}$ Voy. BARTHES Roland, Dans le bruissement de la langue, Paris, Le Seuil, 1984 ; et du même auteur, "Le discours de l'histoire ", in Barthes Roland, Euvres complètes, t. 2, Paris, Le Seuil, 1994, pp. 417-427.

${ }^{24}$ Certeau Michel de, "L'opération historique », in LE Goff Jacques et NORA Pierre (dir.), Faire de l'histoire, t. 1, «Nouveaux problèmes », Paris, Gallimard, 1974, pp. 19-68.

${ }^{25}$ VeYne Paul, Comment on écrit l'histoire, Paris, Le Seuil, 1971, p. 111.
} 
faux problèmes. Comme le roman, l'histoire trie, simplifie, organise, fait tenir un siècle en une page et cette synthèse du récit est non moins spontanée que celle de notre mémoire quand nous évoquons les dernières années que nous avons vécues ${ }^{26}$.

Pour autant, si la question des liens entre le juge et l'historien se révèle relativement ancienne, on ne saurait omettre le fait qu'elle a pris, depuis les années 1970, une dimension nouvelle, dont l'adoption du régime de l'imprescriptibilité des crimes contre l'humanité et l'entrée sur la scène judiciaire d'une partie des spécialistes de l'histoire du temps présent constituent les marques les plus tangibles.

Avec l'ouverture des premiers procès pour crimes ou complicité de crimes contre l'humanité, le juge s'est en effet trouvé en position d'exercer son métier hors de son temps. On lui assigne désormais la mission d'explorer des faits inscrits dans un contexte qui peut lui apparaître opaque et qui, comme le rappelait le procureur général, Pierre TRUCHE, lors du procès BARBIE, est, dans le même temps, partie prenante de l'infraction de crime contre l'humanité ${ }^{27}$. Or, en dépit de ce décalage, il doit, comme le rappelle Denis SALAS, juger, sans droit à l'erreur, des actes perpétrés dans le cadre d'une conjoncture révo$\operatorname{lue}^{28}$. D'où la nécessité pour lui de recourir de plus en plus régulièrement au savoir spécifique des historiens afin d'éclairer une période historique qu'il ne maîtrise pas ou peu; en d'autres termes, d'expliciter un contexte. "L'imprescriptibilité, singularité radicale des procès pour crime contre l'humanité, soulignent Liora ISRAËL et Guillaume MOURALIS, rend presque nécessaire l'intervention des historiens (compte tenu de la césure entre le temps des faits et celui du jugement), ce qui ne veut pas dire qu'elle est légitime $»^{29}$.

Ce changement d'échelle dans les prérogatives du juge explique que l'historien ait été amené à déplacer ou élargir le territoire de ses pratiques professionnelles: l'histoire, plus qu'auparavant, s'est vue investir - parfois, malgré elle - le prétoire.

\footnotetext{
${ }^{26}$ VeYne Paul, Comment on écrit l'histoire., pp. 14-15.

${ }^{27}$ Pierre TRUCHE justifiait la présence d'historiens dans le procès de la façon suivante : «Si on doit entendre aujourd'hui ces témoins d'intérêt général, c'est parce que l'on se trouve dans le cadre de crimes contre l'humanité. [...] Le crime contre l'humanité, c'est quelque chose qui a fait suite à un complot, la Cour de cassation dit "politique d'hégémonie idéologique », c'est-à-dire qu'il faut que j'établisse que ce que Barbie a fait à Lyon, il l'a fait au nom d'une doctrine de l'Etat nazi et qui visait à imposer sa main mise sur une population et qui visait à les décimer. Alors, dans cette mesure-là, les témoins d'intérêt général qui viennent vous dire ce qu'est le nazisme, c'est vraiment le procès ", voy. Audition n 17, https://www.histoire.fr/jaune/html/evenements.htm. ${ }^{28}$ Salas Denis, "La justice entre histoire et mémoire ", in JEAN Jean-Paul et SALAS, Denis (dir.), Barbie, Touvier, PAPON. Des procès pour la mémoire, Paris, Autrement, 2002, p. 22 (20-34).

${ }^{29}$ ISRAËL Liora et MOURALIS Guillaume, "Le chercheur en sciences sociales comme acteur du procès », Droit et société, n44-45, 2000, p. 164 (159-175).
} 
La nouveauté, si ce n'est l'ampleur du phénomène, ne réside pas ici, on l'a dit, dans le fait que les historiens puissent intervenir dans le cadre judiciaire. Elle se comprend en revanche, à travers le rôle qui, dans ce cadre, leur revient. Alors que, dans les périodes antérieures, celuici intervenait au nom de son expertise en écritures ${ }^{30}$ ou en vertu de sa connaissance aiguë des dépôts d'archives, il doit désormais, dans le cadre des procès pour crime contre l'humanité, dépasser ce rôle proprement technique pour exposer à la cour un contexte historique général. Au cours du procès PAPON, Robert $O$. PAXTON et Henri AMOUROuX ont ainsi été chargé de retracer les circonstances globales de l'Occupation, puis Jean-Pierre AzÉMA est venu exposer les caractéristiques de la Révolution nationale, Philippe BURIN, la collaboration d'Etat, Marc-Olivier BARUCH la situation de l'administration, tandis que René RÉMOND évoquait l'antisémitisme ${ }^{31}$. Or, dans la perspective du régime de l'imprescriptibilité, le contexte apparaît, en luimême comme constitutif du crime. Dès lors, l'historien ne se retrouve plus seulement en position d'éclairer la cour mais son savoir participe directement de la définition du crime et donc du jugement.

\section{De l'impossibilité juridique de l'expertise aux impasses du témoignage}

L'une des questions qui se posent, en premier lieu, a trait au statut juridique de l'historien dans le prétoire et dont le procès de Maurice PAPON a notamment révélé qu'elle pouvait placer l'historien dans une position de relative fragilité : celle, pour reprendre l'expression de Jacques WALTER et Béatrice FLEURY-VILATTE, du " témoin-expert " ${ }^{32}$.

Lors du procès de Bordeaux, les historiens n'ont en effet pas été cités à comparaître à titre d'experts. Hormis des cas bien spécifiques, le Code de procédure pénale français n'envisage que deux configurations possibles en matière d'expertise. Soit l'expert convoqué par une cour de justice entreprend, à l'instar des experts en balistique ou en génétique, une performance d'ordre strictement technique et rend compte de phénomènes établis par des lois scientifiques et supposées, à ce titre, reproductibles en toute circonstance. Il peut alors éclairer la cour quand bien même il ne connaît pas le cas jugé. Soit,

\footnotetext{
30 Voy. par exemple RıBÉMONT Thomas, "Les historiens chartistes au cœur de l'affaire Dreyfus ", Raisons politiques, n¹8, 2005, pp. 97-116.

${ }^{31}$ Ce dernier s'est d'ailleurs montré réticent à déposer sur ce point considérant qu'il n'en était pas spécialiste; ce qui pose le problème des critères fondant une expertise. 
comme dans le cas d'expertises psychiatriques, il connaît l'accusé et/ ou a eu accès au dossier versé à la cour, ce qui lui permet de rendre un diagnostic

Or, les historiens cités à comparaître dans le cadre du procès PAPON ne pouvaient, compte tenu de leur position et de la nature des connaissances qu'ils produisent, être dans une de ces deux positions : pas plus qu'ils n'étaient en mesure d'invoquer des lois universelles, ils ne pouvaient, sauf exception, prétendre connaître le dossier. Seul, parmi les historiens, encore qu'il ne s'agisse pas de professionnels de l'histoire au sens strict du terme, Michel BERGÈs, à l'origine, avec Michel SLITINSKY et Gérard BOULANGER, des poursuites contre l'ancien haut fonctionnaire, et Jacques DELARUE ${ }^{33}$ avaient préalablement pu apprécier les pièces du dossier.

Néanmoins, en dehors de ces cas spécifiques, les historiens appelés à la Cour d'assises de la Gironde ne connaissaient ni le dossier, ni l'accusé. Robert O. PAXTON, dans sa déposition du 31 octobre 1997, précisera d'ailleurs d'emblée n'avoir jamais travaillé sur les archives départementales de la Gironde. "Je ne suis ni un spécialiste de la Gironde sous l'Occupation ni de la carrière administrative de M. PAPON ${ }^{34}$.

De surcroît, en raison de la nature même de la discipline historique et de ses objets, les historiens ne peuvent rendre compte de phénomènes reproductibles, tels que ceux habituellement exposés dans le cadre d'expertises techniques. Tout juste sont-ils en mesure de souligner des récurrences tant «les régularités de l'histoire, écrit Antoine PROST, ne peuvent être énoncées que sous la réserves 'toutes choses égales par ailleurs', et les choses ne sont jamais égales, seule-

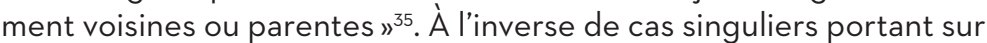
l'authentification de documents ou d'écritures et des rares cas d'expertises historiennes en cours d'instruction, leur intervention ne peut donc a priori s'inscrire dans le cadre juridique d'une expertise. Enfin, l'examen des questions techniques suppose que l'expert se garde de

\footnotetext{
${ }^{33}$ Le cas de Jacques DeLARUE est ici problématique. Nommé avec André GouRON (qui déposera le même jour que lui) lors de la première instruction pour mener une expertise sur les pièces d'archives de la Préfecture de la Gironde - instruction annulée en 1987 -, ce dernier n'est pas un historien professionnel (André Gouron est quant à lui professeur à la Faculté de droit). Il est commissaire divisionnaire honoraire à la retraite. Néanmoins, ses travaux, en particulier son Histoire de la Gestapo, traduite en dix-huit langues, sont reconnus par nombre d'historiens professionnels. En outre, il est collaborateur régulier du Centre de documentation juive contemporaine au sein duquel il entreprend des travaux d'ordre historique. On touche là à la question épineuse de la porosité des frontières entre l'amateur et le professionnel.

34 Erhel Catherine, Aucher Mathieu et De La Baume Renaud (textes réunis par), Le procès de Maurice PAPON, 8 octobre 1997 - 8 janvier 1998, compte-rendu sténographique, Paris, Albin Michel, collection "Les grands procès contemporains ", T.1, 1998, p. 307.

35 Prost Antoine, Douze leçons sur l'histoire, Paris, Le Seuil, 1996, p. 288.
} 
toute problématisation en termes de valeurs et qu'il ne fasse pas d'exposé portant sur la qualification juridique des faits, cette prérogative ne pouvant nullement être déléguée. D'où le paradoxe que relevait Yan THOMAs dans Le Débat : comment les historiens peuvent-ils échafauder " une interprétation générale de Vichy, de sa politique, de son régime, de la responsabilité de ses élites administratives, sans un minimum d'évaluations éthiques (...) $\|^{36}$. Peut-on " demander à un historien de Vichy d'exposer ce qu'il sait et pense d'un tel régime sans lui demander en même temps de répondre d'une manière plus ou moins claire à la question de sa nature - du point de vue notamment de ce que la jurisprudence appelle maladroitement 'un État pratiquant une politique d'hégémonie idéologique' " ${ }^{37}$. Avec le procès TOUVIER, on a pu mesurer les impasses auxquelles pouvait mener cette disjonction entre la qualification juridique des faits et leur étude selon les règles de la méthode historique. La définition jurisprudentielle insiste, en effet, sur la nature systématique et concertée du crime contre l'humanité et limite ce dernier, sur la base de l'arrêt de la chambre criminelle de la Cour de cassation du 20 décembre 1985, aux actes commis au nom d'un Etat pratiquant une politique d'hégémonie idéologique. Or, durant le procès de Versailles, il a été considéré que cette notion ne pouvait s'appliquer au régime de Vichy. Aussi, a-t-il fallu juger le massacre de Rillieux sous l'angle de la complicité avec les autorités nazies. Pourtant, il s'agissait d'un crime perpétré non sur l'injonction des Allemands, mais sur la base d'un règlement de compte entre la Résistance et la Milice françaises. En définitive, en condamnant Paul TOUVIER à perpétuité pour intelligence avec l'Allemagne nazie, la cour d'assises de Versailles a rendu un verdict dont il n'y a pas lieu de contester la légitimité morale, mais qui constitue un arrangement avec les documents et les connaissances historiques disponibles sur l'affaire de Rillieux. Comme nous l'avons déjà souligné, " on touche là (...) à l'un des problèmes les plus épineux concernant les rapports qu'entretiennent la justice et l'histoire. Car, ce qui trouble est de constater la possibilité d'un tel verdict alors même qu'avaient été auditionnés lors de ce procès des historiens de renom ${ }^{38}$, et, en particulier, les membres de la commission René RÉMOND chargés par le Cardinal DECOURTRAY de faire la lumière sur les rapports de l'Eglise catholique avec Paul TOUVIER. Ces historiens ont vu leur compte-rendu servir un [jugement] qu'ils ont perçu rétrospectivement comme un travestissement de la

\footnotetext{
${ }^{36}$ Thomas Yan, "La vérité, le temps, le juge et l'historien ", Le Débat, n 102, décembre 1998, p. 31. 37 Ibid., p. 31.

${ }^{38}$ Les historiens présents au procès PAPON ont tous été cités comme témoins à charge : René RÉMOND par l'avocat général ; François BÉdARIDA, Michel ChanAL, Robert O. PAXTON par les parties civiles. Voy. sur ce point, BÉDARIDA François (dir.), Touvier. Le dossier de l'accusation, Paris, Le Seuil, 1996.
} 
vérité historique et ont parfois vu leurs conclusions "récupérées ", notamment par les parties civiles, alors même qu'ils avaient unanimement refusé de se prononcer sur la culpabilité de Paul TOUVIER ${ }^{39}$.

Pour des raisons juridiques, les historiens ont donc déposé à la cour d'assises de Versailles et à celle de Gironde non en qualité d'experts mais comme témoins. Cette dernière posture pose, elle aussi, des problèmes et il n'est pas certain qu'elle ait permis aux historiens de s'inscrire pleinement dans le temps de l'histoire. Le statut de témoin implique de déposer oralement et sans note. De plus, ce qui constitue la matière d'un témoignage repose sur la mémoire. "C'est pourquoi, note Yan THOMAS, l'oralité est une règle de fond du témoignage pénal ou civil. Elle n'est pas une forme surimposée à un savoir extérieur à elle. La forme est ici constitutive et garante du fond. La parole véhicule l'expérience de celui qui parle. Ainsi s'explique l'interdiction faite aux témoins de s'aider de documents écrits. Les dépositions écrites ne valent que pour les témoins non comparants (l'écrit représentant ici la parole), ou pour ceux qui ont déjà achevé leur déposition orale en cours d'instruction. Tout cela fait du procès un événement où de la mémoire prend forme. De la mémoire, pas de l'histoire ${ }^{40}$. Or, les historiens ne sont pas - du moins est-ce rarement le cas - des témoins oculaires ${ }^{41}$ et leur travail nécessite généralement le support de l'écrit. Dès lors, comment comprendre le statut de témoin qui a été le leur durant les procès TOUVIER et PAPON. Certains juristes ont avancé que cette posture pouvait être légitimée par la notion d'imprescriptibilité de crime contre l'humanité. En effet, en définissant une temporalité immobile, le régime d'imprescriptibilité nous rend tous contemporains des crimes passés dont les coupables sont encore vivants. Á ce titre, les historiens ont pu être considérés comme des témoins privilégiés. La durée d'un procès, c'est donc mis en place un processus d'hybridation entre le temps du droit, qui n'est autre qu'une construction réglementaire, et le temps de l'histoire. Cette ambivalence du témoignage

\footnotetext{
${ }^{39}$ RıBÉMONT Thomas, op. cit., p. 77. René RÉMOND écrivait d'ailleurs en préambule du rapport intitulé Touvier et l'Eglise : "Il était évident que l'enquête ne porterait pas sur les faits qui avaient motivé les deux condamnations à mort de Paul Touvier et qui font l'objet de l'instruction judiciaire en cours. On ne s'étonnera donc pas que nous ne nous prononcions pas sur sa culpabilité présumée ou son innocence revendiquée. Nous n'avons ni compétence pour le faire ni les moyens de procéder à l'examen des accusations portées contre lui. Nous ne sommes ni des accusateurs ni des avocats ni davantage des juges. C'est à l'instruction d'établir les faits, à l'accusation de fonder son réquisitoire, à la défense d'en prouver l'inanité, au procès public de faire toute la lumière ", in RÉMOND René, AzÉMA Jean-Pierre, BÉDARIDA François, CHOLVy Gérard, COMTE Bernard, Dujardin Jean, Durand Jean-Dominique et HILAIRE Yves-Marie, Paul Touvier et l'Eglise, op. cit., p. 14.

40 Thomas Yan, op. cit., p. 30.

${ }^{41}$ Sur cette notion, voy. DULONG Renaud, Le témoin oculaire. Les conditions sociales de l'attestation personnelle, Paris, Éditions de l'EHESS, 1998.
} 
historique ${ }^{42}$ ne pallie cependant pas les risques évidents d'anachronismes que charrie avec lui le régime d'imprescriptibilité. Durant le procès PAPON, bien que la plupart des historiens présents aient cherché à ancrer leur intervention dans le temps de l'histoire ${ }^{43}$, la question est restée problématique, et ce d'autant plus que leurs dépositions s'inscrivaient dans un agenda qu'ils n'avaient pas eux-mêmes déterminé. L'article 331 du Code de procédure pénale précise, en effet, que «les témoins déposent séparément l'un de l'autre, dans l'ordre établi par le président ". Pour cette raison, on a vu se succéder, de façon très rapprochée, des témoignages portant sur des périodes historiques différentes, ouvrant la brèche à de possibles amalgames ou anachronismes.

On peut, par exemple, se demander dans quelle mesure la déposition de Jean-Luc EINAUDI, qui intervenait seulement quinze jours avant celles des premiers historiens et qui portait sur une période postérieure à celle des faits incriminés n'a pas poussé les jurés à opérer des liaisons anachroniques. En premier lieu, en raison de l'utilisation, dont la légitimité n'a pas à être ici discutée, de termes ayant une forte résonance dans la mémoire de la Seconde Guerre :

Un premier couvre-feu fut alors décrété par M. PAPON, couvre-feu qui s'imposait aux travailleurs nord-africains. Ce couvre-feu tomba rapidement en désuétude. L'action de M. PAPON, à partir de ce moment, s'orienta dans le sens d'une répression collective dirigée contre l'ensemble d'une population visée, au travers de rafles qui se multiplièrent. Ces rafles étaient basées sur l'apparence physique des gens, sur le faciès. En 1959 fut ouvert, en plein bois de Vincennes, un camp qui s'appelait "centre d'identification et de vérification ", où étaient conduites les personnes raflées à Paris et dans la banlieue parisienne. [...] Ces rafles s'accompagnèrent de plus en plus souvent de violences, de mauvais traitements, de sévices. On vit apparaître ce que les policiers appelaient eux-mêmes la pratique des comités d'accueil. Lorsque les personnes raflées en fonction de leur apparence descendaient des cars, elles étaient systématiquement frappées. C'était cela les comités d'accueil. M. PAPON avait alors le pouvoir de faire interner des gens

\footnotetext{
${ }^{42}$ Faute de mieux, nous retenons cette expression mais elle nous paraît peu convaincante puisque le témoignage, en tant que tel, relève plus de la mémoire que de l'histoire.

${ }^{43}$ Lors du procès PAPON, Jean-Pierre AzÉMA a notamment déclaré que s'inscrirait, autant que possible dans le temps de l'histoire. "Je ne suis pas en âge de fournir un témoignage proprement dit, a-t-il souligné. On m'a demandé de faire une déposition. Je la ferai en historien, sans haine, et en évitant (...) l'anachronisme qui, pour un historien, est toujours discutable, dès lors que cet anachronisme peut se mêler au balancier de la mémoire ", voy. ERHEL Catherine, AUCHER Mathieu et DE LA BAUME Renaud (textes réunis par), Le procès de Maurice PAPON, 8 octobre 1997 - 8 janvier 1998, compte-rendu sténographique, op. cit., p. 339.
} 
dans des camps. Le commissaire des Renseignements généraux, à l'époque Paul Roux, m'a indiqué que M. PAPON donnait des ordres d'internement en blanc et qu'il fallait faire du chiffre ${ }^{44}$.

En second lieu, l'évocation de lieux éminemment symboliques pour la mémoire juive et la mention de l'année 1942 ont contribué à établir un lien direct entre Vichy et la période de la guerre d'Algérie. En effet, évoquant le passé de Maurice PAPON comme préfet de police de Paris, ce dernier a souligné :
Á la fin d'août 1958, il ordonnait des rafles de travailleurs nord-africains comme il le dit dans un communiqué. Savez-vous ou M. PAPON les fait interner? Il les fait interner au Vel' d'Hiv, qui existe encore, ainsi qu'à la salle Japy, deux lieux où les juifs avaient été internés avant d'être déportés vers les camps d'extermination nazis. Visiblement, ça ne pose aucun problème de conscience à M. PAPON ${ }^{45}$.

Plus loin, il rappelle que le 17 octobre 1961,

\begin{abstract}
Des milliers de personnes ont été raflées. Comme les véhicules de la police ne suffisent pas, M. PAPON a réquisitionné des autobus de la RATP. En 1942, ce sont les autobus de la TCRP qu'on avait réquisitionnés. Et puis sont réquisitionnés également un certain nombre de lieux : le palais des sports, à la porte de Versailles - le Vel' d'hiv n'existant plus à ce moment -, le stade de Coubertin notamment. [...] II y eut [...] une véritable chasse à l'homme, en fonction du faciès, un massacre commis sous la responsabilité de Maurice PAPON. Des hommes, sous les ordres de Maurice PAPON, qui ont déshonoré la police. Au minimum, deux cents morts, vraisemblablement trois cents ${ }^{46}$.
\end{abstract}

Les thèses développées par Jean-Luc EINAUDI ont pu contribuer à opérer un raccourci historique dans l'esprit des jurés. Si, en 1961, Maurice PAPON a pu ordonner le massacre par la police de ceux cents ou trois cents personnes sur la base de critères raciaux - au faciès -, en faisant enfermer ces derniers au Vélodrome d'Hiver et à la salle Japy après avoir réquisitionné les bus de la RATP, alors ce même Maurice PAPON a, sans doute, été amené à en faire de même en 1942 et 1943.

Lors des questions posées au témoin, on voit d'ailleurs clairement se dessiner, sans pour autant être toujours explicité, le parallèle entre les deux périodes.
Me Michel Tubiana [à Jean-Luc EINAUDI] : Ma question au témoin a trait à la période de Constantine. M. PAPON, hier, nous a indiqué avoir eu connaissance de dérapages, d'excès de l'armée française et en avoir rendu compte à ses chefs. D'après vos recherches, d'après les élé-

\footnotetext{
${ }^{44}$ Erhel Catherine, Aucher Mathieu et De La Baume Renaud (textes réunis par), Le procès de Maurice PAPON, 8 octobre 1997 - 8 janvier 1998, compte-rendu sténographique, op. cit., p. 229.

45 Ibid., p. 227.

46 Ibid., p. 232.
} 
ments portés à votre connaissance, M. PAPON avait-il un autre pouvoir que celui de rendre compte à ses supérieurs des exactions dont il a été établi qu'il avait connaissance? Avait-il pouvoir d'intervenir sur ces exactions?

J.-L. ENAUDI : Concernant cette période, la responsabilité de M. PAPON existe. Je pense qu'elle existe pour tout homme à tout moment. Concernant la torture, vous savez, il y a des militaires qui ont démissionné par refus de la torture. Mais il faut dire une chose, c'est que les supérieurs de M. PAPON durant cette période, et je pense particulièrement à Robert LACOSTE, à Maurice BOURGĖS-MAUNOURY, ministre de la Guerre, à Max LEJEUnE, secrétaire d'Etat à la Guerre, eh bien les supérieurs de M. PAPON ont encouragé l'usage de la torture, ont donné des ordres oraux pour que la torture soit employée lors des interrogatoires. M. PAPON a agi à ce moment dans le cadre d'un système qu'il pouvait refuser, mais il ne l'a pas fait.

Me TuBiana : Avait-il un pouvoir réel d'intervention sur les tortures dont il s'est fait l'écho hier, a contrario, bien entendu?

J.-L. ENAUDI : Je pense qu'il avait un pouvoir d'intervention mais, encore une fois, ce pouvoir d'intervention, pour qu'il le mette en $œ u v r e$, il aurait fallu qu'il entre en conflit avec ses supérieurs et singulièrement avec Robert LACOSTE. Cela fait penser à d'autres débats. Quand on pense être confronté à l'inacceptable, est-ce que l'on peut démissionner? Est-ce que l'on doit démissionner? II y en a qui ont répondu par l'affirmative, d'autres non et qui, par là même, ont pratiqué l'inacceptable ${ }^{47}$

La corrélation entre les deux périodes historiques - celle de Vichy et celle de la guerre d'Algérie -, si elle apparaît en filigranes dans les propos tenus par Jean-Luc EINAUDI est aussi le produit de l'agenda suivi dans l'arène judiciaire. Les parties civiles et les procureurs généraux ne pouvaient en effet ignorer, dès le 16 octobre, qu'interviendrait le 5 novembre suivant l'historien Marc-Olivier BARUCH dont la thèse avait mis en lumière les marges de manœuvre existant au sein de l'administration sous le régime de Vichy. Orienter les questions posées au témoin en ce sens, c'est-à-dire en établissant un pont entre deux conjonctures qui, si elles avaient vu Maurice PAPON détenir d'importantes prérogatives, ne se révélaient pas moins fort différentes, participait d'une des formes possibles de l'instrumentalisation des historiens. "Les magistrat de Bordeaux, écrit Jean-Noël JEANNENEY, peuvent bien rappeler obstinément, dans le droit fil de leur vocation, qu'on ne juge 
en Maurice PAPON que ses actes commis sous l'Occupation. II n'empêche que la suite de sa carrière ne cesse pas de marquer implicitement les esprits ${ }^{48}$.

Pourtant, en droit, le jugement doit concerner, de façon exclusive, les faits contenus dans l'acte d'accusation et doit se concentrer sur les actes entrant dans le champ d'inculpation et non sur le futur de l'accusé. L'historien doit donc en principe borner son intervention à situer le contexte des seuls faits incriminés. Maître VARAUT, pour la défense - bien qu'ayant lui-même recours à ce type de stratégies - soulignera d'ailleurs l'artifice employé par les parties civiles et le ministère public : "Je dois dire que la défense, en ma personne, se reproche de n'avoir pas prévu l'importance que pourrait prendre le procès dans le procès $»{ }^{49}$.

Cette restriction du champ des faits abordables dans une cour d'assises, et qui repose sur l'interdiction de mettre en relation les faits jugés avec d'autres faits - analogues ou non - ne faisant pas partie de la saisine de la cour, demeure néanmoins problématique au regard de la démarche historique. Celle-ci, en effet, induit généralement une réflexion sur les «contextes emboîtés », c'est-à-dire sur la comparaison. Or, dans le procès PAPON, aucune approche comparée des administrations préfectorales n'a été véritablement esquissée alors qu'elle aurait pu servir de point d'ancrage à l'analyse du contexte. Et lorsqu'elle s'aventure sur le terrain du comparatisme, la justice tend à privilégier, comme le montre l'audition de Jean-Luc EINAUDI, les comparaisons d'ordre temporel (de type hier / aujourd'hui) plutôt que celles reposant sur des différences spatiales (de type hier dans un espace $\mathrm{x} /$ hier dans un espace y). Tel n'est d'ailleurs pas le propre des procès pour crimes contre l'humanité. Jean-Noël JEANNENEY a ainsi souligné comment, au cours du procès du sang contaminé, l'acte d'accusation luimême se fondait sur une lecture anachronique des faits ${ }^{50}$.

Á travers ces différents exemples, qui ont pour la plupart trait aux normes procédurales du témoignage, on comprend que le statut des historiens dans les procès précités soit apparu peu compatible avec le discours historique rendant a priori difficile la manifestation de la vérité historique au sein du prétoire. En dépit de la formule sacramentelle propre au témoignage de «dire la vérité, toute la vérité, et rien que la vérité ", le président du tribunal, Jean-Louis CASTAGNÈDE rappelait d'ailleurs que "l'interrogatoire n'est pas destiné à établir la vérité

\footnotetext{
${ }^{48}$ JeAnNeney Jean-Noël, Le passé dans le prétoire. L'historien, le juge et le journaliste, Paris, Le Seuil, 1998, p. 73.

${ }^{49}$ ERhel Catherine, Aucher Mathieu et De La Baume Renaud (textes réunis par), op. cit., T. 1, 1998, p. 240.

50 JeAnNEnEY Jean-Noël, "Un monstre dans le prétoire : l'anachronisme ", Le Monde, 22 janvier 1999, p. 13.
} 
mais à permettre à la Cour et aux jurés de se faire une opinion $»^{51}$. La recherche de la réalité des faits constituerait donc un objectif secondaire. Les preuves, comme le souligne Xavier LAGARDE à propos du juge civil, participeraient essentiellement d'une logique de légitimation du jugement sous l'apparence de règles attestant la recherche objective de la réalité ${ }^{52}$. D'où, pour beaucoup d'historiens, à l'instar d'Henry Rousso, une disjonction entre le discours historique et la pratique judiciaire, disjonction d'autant plus grande que, à ses yeux, dans le cadre pénal, l'historien n'est pas maître des questions qu'il se pose ${ }^{53}$, pas plus qu'il ne peut revenir sur ces conclusions ${ }^{54}$. Henry Rousso note, par exemple, que les historiens présents lors du procès PAPON n'ont pu développer spontanément la question de la signification des actes de résistance de la part de fonctionnaires par ailleurs coupables d'actes criminels antérieurs. Or, ce point se révélait essentiel pour comprendre la fin du régime de Vichy, mais aussi le cas spécifique de Maurice PAPON. Il y aurait eu là une forme d'auto-instrumentalisation à rebours des historiens puisque, d'une certaine façon, évoquer cette question « aurait été servir, dans la logique des assises mais pas dans la logique historienne, la cause de la défense $»^{55}$. En outre, on voit bien qu'à la démarche historienne risque de se substituer, non seulement celle du juge, mais aussi celle qui sous-tend des mémoires souvent concurrentes.

\section{Quand l'historien endosse les habits du juge}

Lors des procès TOUVIER et PAPON, la plupart des historiens sont intervenus avec pour objectif de fournir à la cour le contexte se rattachant aux faits incriminés. Cette démarche s'est avérée nécessaire aux juges dans la mesure où la durée séparant les actes justiciables du procès se révélait particulièrement longue. À propos du procès PAPON, écrit Jean-Noël JEANNENEY, «c'est [en définitive] comme si on

\footnotetext{
${ }^{51}$ Cité dans Conan Éric, "Les deux vérités », L'Express, 29 janvier 1998.

${ }^{52}$ Voy. sur ce point, la thèse défendue par LAGARDE, Xavier, "Vérité et légitimité dans le droit de la preuve ", Droits, n²3, 1996. Ce point doit toutefois être nuancé : si pendant longtemps, le juge civil n'a pas eu pour objectif premier la recherche de la vérité, l'article 10 du Code civil lui impose néanmoins désormais d'en faire une priorité.

${ }^{53} \mathrm{Or}$, comme le rappelait Marc BLOCH, la construction libre du problème est au centre de l'opération historique.

${ }_{54}$ Alors que le travail d'expertise produit un rapport écrit, sur lequel l'expert peut, en cours d'élaboration revenir, et dont il peut dans le même temps faire discuter les thèses. En outre un rapport d'expertise peut susciter une contre-expertise, ce qui, a priori, épouse mieux les canons de la recherche scientifique fondé sur le principe de falsifiabilité. Voy. sur ce point, POPPER Karl, La logique de la découverte scientifique, Paris, Payot, 1954. 
avait jugé Ravaillac en 1665, l'affaire du Collier de la reine au milieu de la monarchie de juillet, le duc d'Enghien à la fin du second Empire, les responsables de Panama à la Libération, EsterhaZY au temps du gouvernement de Pierre MENDÈs FRANCE ou encore les mutins de 1917 après mai $1968 \|^{56}$. Interrogé par Maître VARAUT sur le rôle de l'historien, Robert O. PAXTON justifiera d'ailleurs sa présence au procès PAPON de la façon suivante:

\begin{abstract}
Je crois que les historiens peuvent avoir un rôle. Ma vie aurait sans doute été plus simple si j'avais refusé. Un historien n'est pas un témoin oculaire, il ne juge pas. Mais il a un rôle bien précis à jouer dans un procès. Je prendrai comme exemple le procès TOUVIER : les historiens ont témoigné et leur rôle n'était pas de parler de ce qu'ils avaient vu mais de fournir le contexte, fondé sur une documentation aussi complète que possible à la lumière de laquelle certaines affirmations ne sont plus possibles tout comme certaines explications deviennent plus claires. Les historiens ont le rôle de situer, décrire le contexte des faits; ils ont un rôle authentique et je l'ai accepté ${ }^{57}$.
\end{abstract}

Pour autant, et bien qu'il s'y refuse, l'historien peut, en raison même du rôle qu'on lui assigne, être entraîné à revêtir, malgré lui, les habits du juge. En effet, lorsqu'il retrace un contexte ce dernier trouve en position de décrire des chaînes de causalité impersonnelles, c'est-àdire des processus collectifs qui constituent la matière même de l'histoire.

\begin{abstract}
Que se passe-t-il, écrit Yan THOMAs, lorsque l'acte (par exemple, avoir accepté de tenir une position d'autorité hiérarchique sur le bureau des questions juives, avoir accepté de servir d'intermédiaire, par sa signature, entre les ordres criminels d'un préfet et leur exécution par la police) ne prend sa signification juridique, au regard du crime contre I'humanité, que dans la mesure où il s'inscrit dans nombre de circonstances proches et lointaines - déportations massives dont l'administration préfectorale est l'instrument, politique systématique d'extermination ou de persécutions raciales dans le plan desquelles cette administration s'aligne, visée d'hégémonie idéologique des Etats qui conçoivent cette politique et font exécuter ce plan ?58
\end{abstract}

Le crime contre l'humanité a ceci de spécifique que l'inclusion du contexte dans l'acte est au centre de sa définition juridique. C'est pourquoi la singularité de l'acte ne prend pas place dans un environnement criminel mais se trouve portée et constituée par cet environnement, alors même que, pour le juge, le contexte ne fournit traditionnellement qu'une circonstance extérieure susceptible d'atténuer

\footnotetext{
${ }^{56}$ JeAnneney Jean-Noël, Le passé dans le prétoire, op. cit., p. 69.

${ }^{57}$ Erhel Catherine, Aucher Mathieu et De LA BAUMe Renaud (textes réunis par), Le procès de Maurice PAPON,

8 octobre 1997 - 8 janvier 1998, compte-rendu sténographique, op. cit., p. 317.

${ }^{58}$ Thomas Yan, op. cit., pp. 33-34.
} 
ou d'aggraver la responsabilité pénale d'un individu, irréductiblement auteur et responsable de ses actes pénalement. Autrement dit, le principe d'individualité de la responsabilité pénale se heurte au fait que le contexte est intrinsèquement constitutif du crime contre l'humanité. De ce point de vue, par son ampleur même, le crime contre l'humanité tend à pervertir le rapport ordinaire du fait à son contexte et implique pour le juge, comme pour l'historien, de les considérer sur un même plan. Á partir du moment où le contexte des actions qu'il s'interdit, a priori, de juger sert précisément à qualifier ces actions comme crimes, I'historien se voit donc presque immanquablement investir le champ du juge.

Subséquemment, l'appel aux historiens professionnels et à leur travail de généralisation présente le risque de faire basculer une responsabilité d'ordre collectif sur la responsabilité d'un individu. Cette logique, bien que largement décriée par les historiens, est d'ailleurs explicitement revendiquée par nombre de porte-parole d'associations de déportés ${ }^{59}$. Serge KLARSFELD, aux yeux duquel il s'agissait, à travers Paul TOUVIER, de juger la Milice et, à travers Maurice PAPON, de faire le procès de l'administration préfectorale, le rappelait avec force : "Le propre de biais judiciaire, souligne-t-il, est de personnaliser. Cela est beaucoup plus efficace que de se promener avec des livres ou des documents historiquement irréfutables (ce que l'on fait parallèlement qui n'ont qu'un nombre limité de lecteurs. Cette personnalisation est le meilleur moyen pour faire passer auprès des médias et du grand public les événements dont les personnages ciblés ont été les acteurs ou les responsables "60. Se présente alors, pour l'historien un triple danger. Soit il s'éloigne de la démarche historienne, « la question de savoir si un secrétaire général de préfecture était ou non un acteur important sous l'Occupation [semblant] en tant qu'historien, très secondaire au regard de la question principale, à savoir le fonctionnement d'une telle administration dans son ensemble ${ }^{6}$. Soit, il contribue à renforcer les partisans de la responsabilité collective, voire ceux du principe du bouc émissaire. Soit, enfin, son exposé général pèse directement sur le verdict ce qui revient à admettre qu'un acteur concentre à lui seul la charge symbolique d'un procès qui, quoi qu'on en dise, est aussi celui d'une époque et d'un régime. Marc-Olivier BARUCH notait ainsi, à propos du procès PAPON, que «l'on

\footnotetext{
${ }^{59}$ C'est d'ailleurs surtout le fait de ce type de collectif. En raison de cette collusion possible entre des échelles différentes, les magistrats français ont été particulièrement réticents à embrasser cette logique. On peut d'ailleurs noter que dans toutes les affaires relatives à la Seconde Guerre mondiale, le ministère public n'a jamais pris l'initiative des poursuites.

${ }^{\circ}$ Cité in Jeanneney Jean-Noël, Le passé dans le prétoire, op. cit., p. 80.

${ }^{61}$ Rousso Henry, La hantise du passé, op. cit., p. 100.
} 
ne pût empêcher que ce procès devienne celui de Vichy à travers le cas de Maurice PAPON ${ }^{62}$. Certains avocats des parties civiles, dans le sillage de l'école dite "fonctionnaliste " ont, par exemple, amplement insisté sur la notion de "crime de bureau " considérant comme criminelle, à un niveau de responsabilité effectif, l'appartenance à une chaîne de décisions ayant participé aux opérations de déportation. Ce type d'analyse, qui suppose que le comportement d'un individu n'est pas détachable du fonctionnement et des valeurs qui régissent le système, en faisant disparaître la notion de responsabilité individuelle, induit d'ailleurs une contradiction avec le droit pénal français. Il n'appartient en effet pas au juge de rechercher l'exonération de la responsabilité dans des causes inhérentes à la société.

Les historiens se sont certes, la plupart du temps, défendus de confondre les genres historique et judiciaire insistant sur l'idée que, en participant à un procès, ils cherchaient avant tout à « expliquer » et non à porter un jugement. Pourtant, rétrospectivement, Robert O. PAXTON a admis que la frontière entre l'explication et le jugement n'était pas aussi étanche que certains des historiens présents au procès PAPON le prétendaient. Or, dans cette perspective, dès l'instant où un procès pour crime contre l'humanité constitue un événement exceptionnel dans lequel se joue une histoire collective - au risque, pour reprendre la formule d'Hannah ARENDT de ne pas se prononcer sur "les seules choses qui comptent lors d'un procès criminel $"^{63}-$, l'intervention des historiens prend inévitablement une résonance politique. "On ne sortira jamais d'une contradiction, affirmait ainsi Henry Rousso à propos du procès de Bordeaux : on a voulu faire un procès de droit commun, mais c'est un procès fondamentalement politique et exceptionnel, jusque dans ces dernières péripéties ${ }^{64}$.

\section{Conclusion}

L'intervention des historiens dans les procès TOUVIER et PAPON, dont on attendait qu'elle ait «valeur de digue quand les dérives de la mémoire peuvent tout envahir ${ }^{65}$ aurait donc, d'une certaine façon, « raté son sujet » en raison des contraintes pesant sur l'historien. Parmi

\footnotetext{
${ }^{62}$ BARUCH Marc-Olivier, "L'historien et la justice ", in RUANO-BORBOLAN Jean-Claude (dir.), L'histoire aujourd'hui, Auxerre, Ed. Sciences humaines, 1999, p. 221.

${ }^{63}$ Notamment, « le problème de la culpabilité ou de l'innocence de l'individu, de la justice rendue à l'accusé et à la victime ", in ARENDT Hannah, Eichmann à Jerusalem. Rapport sur la banalité du mal, Paris, Gallimard, 1966, pp. 325-326.

${ }^{64}$ Rousso Henry, "PAPON n'est pas un détenu ordinaire ", propos recueillis par Antoine DE GAUDEMAR et Pascale NiVELLE, Libération, 19 janvier 2001, p. 5.

${ }^{65}$ Salas Denis, "La justice entre mémoire et histoire ", op. cit., p. 27.
} 
ces dernières, une première tient à la difficulté d'appliquer des travaux historiques à un individu singulier responsable personnellement de ses actes sur le plan pénal. Une autre renvoie au risque d'assujettissement de la recherche aux règles préconstruites du droit et aux modalités propres du questionnement juridique. Enfin, le cadre hétéronome dans lequel prend place «l'expertise/témoignage » de l'historien laisse potentiellement place à la déformation, voire à l'instrumentalisation du discours historique et à une politisation du savoir historien.

Sur le fond, le cœur du problème tiendrait au fait que, en raison de la nature de la scène judiciaire, de ses protocoles et du statut juridique sous lequel ils déposent, les historiens verraient leur autonomie de questionnement limitée et leurs travaux servir des logiques autres que celles en vigueur dans le champ de la recherche. Pourtant, si malaise il y a, celui-ci ne saurait se réduire à la problématique de l'hétéronomie. En particulier, parce que les procès eux-mêmes, compte tenu de leur caractère exceptionnel et de la couverture médiatique dont ils ont fait l'objet, ont suscité, aux marges de l'arène judiciaire proprement dite, d'autres types d'interventions. Michaël MARRUS, Denis PESCHANSKI ou encore Eberhard JAËCKEL ont ainsi largement commenté, au fil des jours, pour ne pas dire "en direct ", les développements du procès PAPON dans les journaux, ce qui, à tout prendre, les plaçait eux aussi dans une configuration normative différente de celle en vigueur dans le monde de la recherche.

Par-delà la question de l'hétéronomie, qui se pose aussi dans le champ académique puisque les historiens constituent un corps rattaché à l'Etat, l'embarras semble essentiellement, résulter de la recomposition de l'univers des pratiques de l'histoire. Les effets d'hybridation entre le champ judiciaire et celui de l'histoire ne s'opèrent pas seulement dans le sens d'un transfert qui irait de l'histoire au judiciaire. La multiplication des ponts entre l'histoire et la justice a aussi des conséquences sur la définition même des pratiques de recherche en histoire comme l'attestent les controverses autour du "génocide vendéen ${ }^{66}$ ou encore du Livre noir du communisme ${ }^{67}$.

Enfin, les procès PAPON et TOUVIER ont reposé de façon aiguë le problème de l'utilisation de l'histoire " savante " en dehors des institutions légitimes que sont l'Université et le CNRS. Ce faisant, ils ont aussi cristallisé certaines des lignes de fracture qui traversent la discipline, et réactualisé des controverses épistémologiques anciennes sur

\footnotetext{
${ }^{66}$ Voy. sur ce point, CLÉMENT Jean-Martin, "A propos du génocide vendéen. Du recours à la légitimité de I'historien ", Sociétés contemporaines, n³9, 2000, pp. 23-38.

${ }^{67}$ Courtols Stéphane, Werth Nicolas, PANNÉ Jean-Louis, PACZkowski Andrzej, Bartosek Karel et Margolin Jean-Louis, Le livre noir du communisme. Crimes, terreur, répression, Paris, Robert Laffont, 1997.
} 
les usages de l'histoire. «Il a toujours existé, écrit François BÉDARIDA en simplifiant l'analyse, une tension entre deux conceptions. L'une prône un savoir désintéressé, sorte d' 'histoire pour l'histoire', à la manière de l' 'art pour l'art' : c'est elle qui a été mise en avant par l'école positiviste et dont on retrouve aujourd'hui de nombreuses traces (pour LANGLOIS et SEIGNOBOS, la connaissance avait une valeur absolue et par elle-même, elle était sans utilité sociale, le but de l'histoire n'étant ni de plaire, ni de donner des recettes, mais seulement de savoir). L'autre conception accorde au contraire à l'histoire une fonction éminente dans la société et ses tenants sont convaincus qu'elle est non seulement utile, mais nécessaire à la collectivité ${ }^{68}$. Cette tension n'intéresse pas seulement l'histoire ; elle est au cœur de préoccupations qui balayent l'ensemble des sciences sociales ${ }^{69}$ et montre combien il est nécessaire de poursuivre la réflexion sur l'hybridation qui tend à se renforcer entre le monde de la recherche et les autres sphères de la société.

\footnotetext{
${ }^{68}$ BÉDARIDA François, "La dialectique passé/présent et la pratique historienne ", in BÉDARIDA François (dir.), L'histoire et le métier d'historien en France 1945-1995, 1995, Paris, Éditions de la MSH, p. 84. Voy. aussi, du même auteur, "Praxis historienne et responsabilité ", Diogène, 168, 1994, pp. 3-8.

${ }^{69} \mathrm{La}$ question de l'expertise a, par exemple, était au cœur des débats sur le PaCS. Certains chercheurs, comme Éric FASSIN, Marcela IACUB, ou encore Daniel BORILLO, ont, par exemple, vivement critiqué l'extension actuelle du "territoire de l'expert ", voyant dans l'expertise une menace pour l'autonomie de la science et la marque d'une subordination croissante de la délibération politique aux normes édictées par les savants. Voy. BorILlo Daniel, FASSIN Éric et IACUB Marcela (dir.), Au-delà du PaCS. L'expertise familiale à l'épreuve de l'homosexualité, Paris, PUF, 1999.
} 